\title{
Paeoniflorin, a Natural Product With Multiple Targets in Liver Diseases-A Mini Review
}

\author{
Xiao Ma ${ }^{1}$, Wenwen Zhang ${ }^{1}$, Yinxiao Jiang ${ }^{1}$, Jianxia Wen ${ }^{1,2}$, Shizhang Wei ${ }^{1,2}$ \\ and Yanling Zhao ${ }^{2 *}$
}

1 School of Pharmacy, Chengdu University of Traditional Chinese Medicine, Chengdu, China, ${ }^{2}$ Department of Pharmacy, Fifth Medical Center of PLA General Hospital, Beijing, China

\section{OPEN ACCESS}

Edited by:

Alejandro Urzua,

Universidad de Santiago

de Chile, Chile

Reviewed by:

Yi Ding,

Fourth Military Medical

University, China

Fengmei Qiu,

Zhejiang Chinese Medical

University, China

*Correspondence:

Yanling Zhao

zhaoyl2855@126.com

Specialty section:

This article was submitted to

Ethnopharmacology,

a section of the journal

Frontiers in Pharmacology

Received: 23 August 2019

Accepted: 03 April 2020

Published: 28 April 2020

Citation:

Ma X, Zhang W, Jiang Y, Wen J, Wei S and Zhao $Y$ (2020) Paeoniflorin, a

Natural Product With Multiple Targets in Liver Diseases - A Mini Review.

Front. Pharmacol. 11:531. doi: 10.3389/fphar.2020.00531
Paeoniflorin is derived from Paeonia suffruticosa Andr., Paeonia lactiflora Pall., or Paeonia veitchii Lynch and has been used in traditional medical applications for more than 2,000 years. Paeoniflorin is a monoterpenoid glycoside with various effects on liver diseases. Recent studies have revealed that paeoniflorin demonstrates a wide range of activities, including hepatic protection, cholestasis alleviation, liver fibrosis attenuation, nonalcoholic fatty liver disease prevention, and hepatocellular carcinoma inhibition involved in multiple pathways. Moreover, anti-inflammation, antioxidation, and immune regulation with the regulation of TLR4-NF- $\mathrm{BB}, \mathrm{ROCK} / \mathrm{NF}-\kappa \mathrm{B}, \mathrm{HO}-1$, mitochondria-dependent as well as HMGB1-TLR4 signaling pathways are correlated with hepatic protection in liver injury and nonalcoholic fatty liver disease. Antioxidative mechanisms, anti-inflammation, and hepatic transporter regulation involved in NOX4, PI3K/Akt/Nrf2, NF-אB, NTCP, BSEP, as well as MRP2 signals are mainly relevant to the anticholestatic effect of paeoniflorin. The inhibition of hepatic stellate cell activation and alleviation of extracellular matrix deposition via vast signals such as mTOR/HIF- $1 \alpha$, TGF- $\beta 1 /$ Smads, and JAK2/STAT6 are primarily involved in the antifibrotic effect of paeoniflorin. The regulation of macrophages also contributes to the alleviation effect on liver fibrosis. In addition, the reduction of invasion, metastasis, and adhesion and the induction of apoptosis-related targets, including Bax, Bcl-2, and caspase-3, are related to its effect on hepatocellular carcinoma. The literature indicates that paeoniflorin might have potent efficacy in complex liver diseases and demonstrates the profound medicinal value of paeoniflorin.

Keywords: paeoniflorin, hepatic protection, cholestasis, liver fibrosis, nonalcoholic fatty liver disease, hepatocellular carcinoma, mini-review

Abbreviations: ALP, Alkaline phosphatase. ANIT, Alpha-naphthylisothiocyanate. BCG, Bacillus Calmette-Guérin. BSEP, Bile salt export pump. $\mathrm{CCl}_{4}$, Carbon tetrachloride. Con-A, Concanavalin A. DMN, Dimethylnitrosamine. ECM, Extracellular matrix. GalN, D-galactosamine. LPS, lipopolysaccharide. GSH, Glutathione. GCLc, Glutamate-cysteine ligase catalytic subunit. GCLm, Glutamate-cysteine ligase modifier subunit. HCC, Hepatocellular carcinoma. HHSECs, Primary human hepatic sinusoidal endothelial cells. HSCs, Hepatic stellate cells. I/R, Ischemia/reperfusion. MRP2, Multidrug resistance-associated protein 2. NAFL, Nonalcoholic fatty liver. NAFLD, Nonalcoholic fatty liver disease. NASH, Nonalcoholic steatohepatitis. NTCP, $\mathrm{Na}^{+}$/taurocholate-cotransporting polypeptide. TBA, Total bile acid. $\gamma$-GT, $\gamma$-Glutamyltranspeptidase. i.g., Intragastric. i.v., Intravenous injection. 


\section{INTRODUCTION}

Since the "one gene, one drug, one disease" concept was challenged, more and more agents have been confirmed to be multiple targets and signals rather than a single approach (Hasin et al., 2017). Several natural products applied for thousands of years in traditional medicine demonstrate a wide range of pharmacological activities via multiple pathways (Park and Pezzuto, 2017; Kunnumakkara et al., 2017; Ren et al., 2019). These natural products might have potent efficacy in complex human diseases and display profound medicinal value. Among these natural compounds, paeoniflorin has gained attention as a promising compound for drug development.

Paeoniflorin is the major bioactive ingredient derived from Paeonia suffruticosa Andr., Paeonia lactiflora Pall., or Paeonia veitchii Lynch, which have been used for cerebrovascular disease, cardiovascular disease, nervous system disease, and liver disease in traditional Chinese medicine for more than 2,000 years (Zhao et al., 2016). Paeoniflorin was first isolated from Paeonia lactiflora Pall. as a monoterpenoid glycoside in 1963 (Zhang J. et al., 2018). Since then, an increasing number of studies have reported the numerous pharmacologic effects of paeoniflorin, such as cerebrovascular protection, cardiovascular protection, neuroprotection, antihyperglycemia, tumor inhibition, immunoregulation, abirritation, and hepatoprotection (Zhang et al., 2017; Chen et al., 2018; Zhai et al., 2018; Tu et al., 2019). Paeoniflorin has gained a large amount of attention for its effect on liver diseases as the growth rate of liver diseases has increased in recent years (Ma et al., 2020). Hence, this mini-review provides a comprehensive summary of the pharmacologic activities of paeoniflorin in liver diseases.

\section{HEPATIC PROTECTION}

The liver is a vital organ for metabolic functions and for the purification of toxic chemicals. However, the liver can be overloaded (Singh et al., 2016). Once the function of the liver is dysregulated, liver damage will occur. Under certain circumstances, liver injuries can be induced by various factors, including chemical pollutants, drugs, alcohols, and liver ischemia (Peralta et al., 2013; Wang et al., 2016; Kullak-Ublick et al., 2017). Liver injury is recognized as a highly complex process accompanied by extensive apoptosis in hepatic cells. Oxidative stress and inflammatory reactions are thought to play key roles in this process (Brenner et al., 2013; Li et al., 2015). Moreover, immune reactions resulting from immune cells such as Kupffer cells have also drawn much attention due to the unique characteristics of hepatic sinusoids (Heymann et al., 2015).

\section{Hepatic Ischemia/Reperfusion Alleviation}

Hepatic ischemia/reperfusion (I/R) injury is the major manifestation after liver transplantation or hemorrhagic shock with relatively high morbidity and mortality (Go et al., 2015). Currently, paeoniflorin is considered to be highly effective in hepatic I/R injury treatment. A study from Xie reported that compared with hepatic I/R injury rats, rats pretreated with paeoniflorin $(100 \mathrm{mg} / \mathrm{kg})$ showed significantly decreased serum alanine aminotransferase (ALT) and aspartate aminotransferase (AST) activities by $40.3 \%$ and $53.8 \%$, respectively. This liver protection effect is strongly relevant to directly alleviating hepatic cell apoptosis and decreasing caspase-3 levels. Furthermore, an inflammatory response was also observed in this study. This study demonstrated that paeoniflorin pretreatment could inhibit CD45+/Ly6G+ neutrophils and the production of proinflammatory cytokines [tumor necrosis factor alpha (TNF- $\alpha$ ) and interleukin-1 beta (IL-1 $\beta)$ ]. Therefore, a reduction in the innate immune response might contribute to this process. This research finally found that inhibiting the HMGB1-TLR4 signaling pathway is the crucial mechanism of paeoniflorin in hepatic I/R injury (Xie et al., 2018). Another study also supported this conclusion. Tao's study demonstrated that treatment with paeoniflorin at a dose of 5 to $20 \mathrm{mg} / \mathrm{kg}$ could markedly reduce the expression levels of inflammatory mediators, including nuclear factor kappa-B $(\mathrm{NF}-\kappa \mathrm{B}), \mathrm{TNF}-\alpha$, IL-1 $\beta$, and IL- 6 . At the same time, the apoptosis marker caspase-3 was decreased after paeoniflorin treatment (Tao et al., 2016). Therefore, antioxidative, antiinflammatory, and antiapoptotic activities are clearly involved in the mechanism of paeoniflorin treatment.

\section{Protection From Toxic Chemical-Induced Liver Injury}

There are a variety of toxic chemicals, including carbon tetrachloride $\left(\mathrm{CCl}_{4}\right)$, concanavalin A (Con-A), Dgalactosamine (GalN), bacillus Calmette-Guérin (BCG), and lipopolysaccharide (LPS), that can induce liver injury (Zhang H.Y. et al., 2018). Toxic chemical-induced liver injury is mainly characterized by an immune response and inflammation. Moreover, hepatic tissue apoptosis is a key outcome. A large amount of evidence indicates that paeoniflorin has a profound effect on toxic chemical-induced liver injury. Paeoniflorin at a dose of $100 \mathrm{mg} / \mathrm{kg}$ was able to decrease liver injury, significantly decrease ALT and AST, and alleviate the histopathological changes induced by $\mathrm{CCl}_{4}$. Moreover, the significant pharmacological effect was related to the reduction of $\mathrm{HO}-1$ mRNA expression and proinflammatory cytokine (TNF- $\alpha$ and IL-6) excretion (Guo et al., 2018). Other studies have indicated the protective effect of paeoniflorin on Con A-induced hepatitis with immune regulation. Chen's results suggested that intravenous paeoniflorin pretreatment could attenuate plasma levels of ALT and AST and diminish apoptosis or necrosis of liver tissue. These results demonstrated a protective effect of paeoniflorin against Con A-induced liver injury in mice. The mechanism may at least in part be the suppression of CD4+, $\mathrm{CD} 8+$, and NKT cell infiltration in the liver. Moreover, the downregulation of TLR4 expression and the inhibition of NF- $\kappa B$ activation are key signaling pathways in this process (Chen $\mathrm{M}$. et al., 2015). In vitro research from primary human hepatic sinusoidal endothelial cells (HHSECs) also confirmed this result. Paeoniflorin at doses from 50 to $800 \mu \mathrm{M}$ most likely contributed to the alleviation of Con-A-induced inflammation in HHSECs. Preincubation with paeoniflorin caused a concentration- 
dependent downregulation of IL-8. Furthermore, paeoniflorin was able to inhibit IL- 8 release by $52.8 \%$ at a dose of $800 \mu \mathrm{M}$. The mechanism might be closely related to blocking IL- 8 secretion via the downregulation of ERK1/2 and Akt phosphorylation (Gong et al., 2015). In addition, GalN/TNF- $\alpha$-induced apoptosis of human L-02 hepatocytes was decreased by paeoniflorin in a dose-dependent manner. The antiapoptotic effect was further evidenced by the inhibition of caspase-3/9 activities and by the suppression of ER stress activation in L-02 cells. These results revealed that paeoniflorin might target ER stress and calcium, leading to mitochondria-dependent pathway regulation (Jiang et al., 2014). A study of immunological liver injury based on BCG combined with LPS was also performed in 2006. Paeoniflorin administration was able to protect against immunological liver injury by ameliorating TNF- $\alpha$ and IL- 6 secretion and downregulating LPS receptor expression (Liu et al., 2006).

\section{CHOLESTASIS ALLEVIATION}

Cholestasis is characterized by decreased bile flow and bile acid accumulation. It is one of the most common but devastating liver diseases. Hepatocyte injury and cholangitis will ultimately occur with cholestasis progression. Furthermore, portal myofibroblast and hepatic stellate cell activation rapidly result in biliary fibrosis or even cirrhosis without prompt treatment (Ghonem et al., 2015). It is currently believed that the pathogenesis of cholestasis involves multiple signaling pathways with the simultaneous activation of inflammation, dysregulation of hepatocyte transporters, and oxidative stress injury in liver tissue (Copple et al., 2010; Allen et al., 2011; Trauner et al., 2017).

A series of studies from Zhao's group indicated that paeoniflorin exerts a dose-dependent $(50-200 \mathrm{mg} / \mathrm{kg})$ protective effect on alphanaphthylisothiocyanate (ANIT)-induced cholestasis in rats by decreasing serum ALT, AST, TBIL, DBIL, total bile acid (TBA), $\gamma$ glutamyltranspeptidase $(\gamma-\mathrm{GT})$, and alkaline phosphatase (ALP). Moreover, the extremely suppressed bile flow induced by ANIT was also increased by paeoniflorin treatment. The mechanism of this activity is partially related to attenuating oxidative stress with reactive oxygen species (ROS) inhibition by suppressing nicotinamide adenine dinucleotide phosphate (NADPH) oxidase 4 expression and the mitochondria-dependent pathway (Zhao et al., 2013; Zhou et al., 2017). In addition, an alternative antioxidative mechanism was also investigated. The results indicated that paeoniflorin could regulate glutathione (GSH) and its related synthase glutamate-cysteine ligase catalytic subunit (GCLc) and glutamate-cysteine ligase modifier subunit (GCLm). The enhancement of GSH synthesis was further proven to increase Nrf2 through the PI3K/Akt-dependent pathway (Chen Z. et al., 2015). Regarding inflammation, histological examination revealed that paeoniflorin-treated rats demonstrated less neutrophil infiltration. The research suggested that paeoniflorin could remarkably reduce the overexpression of NF- $\kappa \mathrm{B}$ and IL- $1 \beta$ induced by ANIT in liver tissue (Zhao et al., 2017). Moreover, a study focusing on Paeonia lactiflora Pall., one of the sources of paeoniflorin, was in accordance with the previous result showing the suppression of the inflammatory response (Ma et al., 2018). Another study further revealed that paeoniflorin could mainly regulate primary bile acid biosynthesis by serum metabolomic profiling analysis (Chen et al., 2016)]. Therefore, transporters might be the central regulatory process. In 2017, Zhao reported that ANITinduced dysregulated hepatocyte transporters, such as $\mathrm{Na}^{+} /$ taurocholate-cotransporting polypeptide (NTCP), bile salt export pump (BSEP), and multidrug resistance-associated protein 2 (MRP2), were restored by paeoniflorin treatment (Zhao et al., 2017).

\section{LIVER FIBROSIS ATTENUATION}

Liver fibrosis is the process of chronic liver injury caused by hepatitis $\mathrm{B}$ and $\mathrm{C}$, alcohol consumption, fatty liver disease, cholestasis, and autoimmune hepatitis (Seki and Brenner, 2015). Hepatic stellate cell (HSC) activation plays a key role in myofibroblasts that produce extracellular matrix (ECM) in the liver (Tsuchida and Friedman, 2017). Currently, a variety of inflammatory and fibrogenic pathways are thought to participate in liver fibrosis (Seki and Schwabe, 2015; Higashi et al., 2017; Wree et al., 2018).

In the $\mathrm{CCl}_{4}$-induced liver fibrosis model, paeoniflorin was proven to effectively attenuate serum ALT, AST, HA, IV-C, and liver tissue Hyp at the doses of 20, 40, 80, and $200 \mathrm{mg} / \mathrm{kg}$ (Min et al., 2011). This result indicated that paeoniflorin could significantly decrease liver fibrosis development. Moreover, the inhibition of HIF- $1 \alpha$ expression partly through the mTOR pathway might be the crucial mechanism (Zhao et al., 2014). This pharmacologic effect was also confirmed in two other liver fibrosis models. Hu reported that paeoniflorin treatment from 20 to $80 \mathrm{mg} / \mathrm{kg}$ for 26 consecutive weeks was able to inhibit radiation-induced hepatic fibrosis. The expression levels of TGF- $\beta 1$, Smad3/4, and Smad7 were significantly lower in the paeoniflorin-treated groups than in the model group. This result indicated that paeoniflorin alleviated fibrosis via the TGF- $\beta 1 /$ Smad signaling pathway ( $\mathrm{Hu}$ et al., 2018). In addition to the radiation model, dimethylnitrosamine (DMN) was also used to induce liver fibrosis. Paeoniflorin treatment demonstrated an antifibrosis effect in rats with less collagen fiber deposition and gentle centrilobular necrosis observed in paeoniflorin-treated rats compared with DMN-induced model rats. These results were at least in part due to restored macrophage disruption and reduced inflammatory cytokines (Chen et al., 2012).

In addition, schistosomiasis is a kind of special chronic disease leading to liver fibrosis. A recent study demonstrated that paeoniflorin at $50 \mathrm{mg} / \mathrm{kg} / \mathrm{d}$ improved parasitological parameters, such as decreased worm burden, immature eggs, and mature eggs, in a schistosomiasis mansoni-induced hepatic fibrosis model. Meanwhile, paeoniflorin treatment also significantly decreased the hepatic mean granuloma diameter and fibrosis area. The mechanism was partially recognized as targeting the apoptosis pathway by regulating caspase-3 and P53 expression (Abd El-Aal et al., 2017). Moreover, the key role of IL13 was also explored in this model. Three other studies confirmed that paeoniflorin had a significant suppressive effect 
on the establishment of the ECM. Paeoniflorin could not only directly inhibit the alternative activation of macrophages by inhibiting the JAK2/STAT6 signaling pathway but also indirectly suppressed macrophages by decreasing IL-13 secretion (Li et al., 2009; Li et al., 2010; Chu et al., 2011).

\section{NONALCOHOLIC FATTY LIVER DISEASE PREVENTION}

Nonalcoholic fatty liver disease (NAFLD) has been the most common chronic liver disease worldwide in recent years. More than $40 \%$ of the population is affected in some countries. NAFLD has attracted concern worldwide since becoming a public health burden (Neuschwander-Tetri et al., 2010; Estes et al., 2018). NAFLD includes a wide range of liver disorders extending from nonalcoholic fatty liver (NAFL) to nonalcoholic steatohepatitis (NASH). Fibrosis, cirrhosis, and hepatocellular carcinoma will ultimately occur without treatment (Lebeaupin et al., 2018).

Paeoniflorin is a potential NAFLD prevention compound according to many studies. Zhang revealed that paeoniflorin attenuated NAFLD by restoring serum ALT, AST, TC, TG, HDL, and LDL. At the same time, paeoniflorin alleviated high-fat dietinduced hepatic adipose infiltration by decreasing steatosis, inflammation, ballooning degeneration, and necrosis. The potential mechanism might be cardiovascular protection by decreasing body weight and hyperlipidemia, blocking inflammation, and inhibiting lipid deposition (Zhang et al., 2015). Further research indicated that paeoniflorin ameliorated hepatic steatosis and inhibited CD68 and TGF- $\beta 1$ expression. Downregulation of the ROCK/NF- $\kappa \mathrm{B}$ signaling pathway might be relevant to the effect of paeoniflorin on NAFLD (Ma et al., 2016). Ma's investigation indicated that $20 \mathrm{mg} / \mathrm{kg}$ paeoniflorin remarkably inhibited lipid ectopic deposition via the lipid metabolism pathway. On the other hand, paeoniflorin treatment also exerted insulin sensitizing effects via IRS/Akt/ GSK3 $\beta$ and antioxidation (Ma et al., 2017). In addition, a recent study also confirmed that paeoniflorin significantly reduced serum insulin and glucagon levels, enhanced insulin sensitivity, restored serum lipid profiles, and attenuated hepatic steatosis. All these effects should be relevant to the activation of the LKB1/ AMPK and Akt signaling pathways in NAFLD (Li et al., 2018).

\section{HEPATOCELLULAR CARCINOMA INHIBITION}

Hepatocellular carcinoma (HCC) is believed to be the most common and malignant type of tumor. HCC is the third most common cancer-related cause of death due to poor prognosis. Over 700,000 HCC cases are diagnosed every year (Robinson et al., 2019). The situation is particularly concerning in China. China accounts for 55\% of HCC cases worldwide ( $\mathrm{Su}$ et al., 2016). HCC is recognized to involve multiple signaling cascades in cell adhesion, cell migration, and extracellular matrix proteolysis (Brown and Murray, 2015). Therefore, potential agents for HCC treatment should efficiently address multiple aspects of this process.

In an in vitro study, paeoniflorin at the doses of 6.25-200 $\mu \mathrm{M}$ was found to significantly inhibit the growth of HepG2 and Bel7402 cell lines. Proteolysis could reduce the invasion, metastasis, and adhesion of HCC cell lines. In addition, paeoniflorin was able to decrease MMP-9 and ERK levels and increase E-cad expression in HepG2 and Bel-7402 cells (Lu et al., 2014). Moreover, another study also indicated paeoniflorin as a promising agent in the treatment of liver cancer. Its mechanism might be partially related to apoptosis induction in hepatocellular carcinoma cells by downregulating prostaglandin $\mathrm{E}$ receptor $\mathrm{EP}_{2}$ levels, increasing the $\mathrm{Bax} / \mathrm{Bcl}-2$ ratio and thus upregulating the activation of caspase-3 (Hu et al., 2013).

\section{OUTLOOK AND CONCLUSION}

As evidenced by the numerous studies that have focused on the mechanism in-depth, many attempts have been made to investigate the efficacy of natural compounds such as paeoniflorin in liver disease treatment. This mini-review summarizes the pharmacologic activities and liver protection provided by paeoniflorin and demonstrates that paeoniflorin from the dosage of $5-200 \mathrm{mg} / \mathrm{kg}$ in vivo is an important compound for hepatic protection, cholestasis alleviation, liver fibrosis attenuation, NAFLD prevention, and HCC inhibition (Table 1). It is also crucial to reveal the mechanism to determine how paeoniflorin exerts its pharmacological effect. Paeoniflorin displays remarkable anti-inflammation effects via the TLR4-NF- $\kappa \mathrm{B}$ and ROCK/NF- $\kappa \mathrm{B}$ signaling pathways during liver injury and NAFLD. Antioxidation signals such as HO-1, mitochondria-dependent pathways, and immune regulation containing HMGB1-TLR4 are closely correlated. Moreover, paeoniflorin also alleviates cholestasis through an antioxidative mechanism by downregulating ROS and NOX4 and upregulating the PI3K/Akt/Nrf2 pathway. The anti-inflammatory effects of NF- $\kappa B$ and IL- $1 \beta$ and the regulation of NTCP, BSEP, and MRP2 are mainly relevant to the anti-cholestatic effects of paeoniflorin. Several important signaling pathways, such as mTOR/HIF- $1 \alpha$, TGF- $\beta 1 /$ Smads, and JAK2/STAT6, are involved in the effect of paeoniflorin on activated HSC and ECM inhibition during liver fibrosis. Macrophage regulation is also considered the crucial mechanism for the antifibrotic effect. The reduction in invasion, metastasis, and adhesion and the induction of apoptosis signals, including $\mathrm{Bax}, \mathrm{Bcl}-2$, and caspase-3, are related to the effect on hepatocellular carcinoma (Figure 1).

The common mechanisms could be summarized from the current literature. The anti-inflammation, anti-oxidative and antiapoptosis in hepatocytes are the core functions for its effect on liver diseases. Moreover, the immune and macrophage regulation are also important for its special effect on liver damage and liver fibrosis. The effect of paeoniflorin on liver diseases has been vastly developed, accompanied by deep insight into mechanistic 
TABLE 1 | The pharmacological activities of paeoniflorin in liver diseases.

\begin{tabular}{|c|c|c|c|c|}
\hline $\begin{array}{l}\text { Disease } \\
\text { Treatment }\end{array}$ & Experimental model & Doses (Route) & Targets/Pathways & Reference \\
\hline \multirow[t]{7}{*}{ Liver injury } & Hepatic I/R-induced injury & 100 mg/kg (i.g.) & HMGB1-TLR4 pathway & Xie et al., 2018 \\
\hline & Hepatic I/R-induced injury & 5-20 mg/kg (i.v.) & NF- $\kappa B$ signaling pathway and caspase- 3 & Tao et al., 2016 \\
\hline & $\mathrm{CCl}_{4}$-induced liver injury & 10-100 mg/kg (i.g.) & HO-1, TNF- $\alpha$, IL-6, and caspase-3 & Guo et al., 2018 \\
\hline & Con A-induced liver injury & $50 \mathrm{mg} / \mathrm{kg}$ (i.v.) & TLR4-NF-кB pathway & Chen M. et al., 2015 \\
\hline & Con A-treated HHSECs & $50-800 \mu \mathrm{M}$ (in vitro) & ERK1/2 and Akt phosphorylation & Gong et al., 2015 \\
\hline & GalN/TNF- $\alpha$-treated LO2 & $1-100 \mu \mathrm{M}$ (in vitro) & ER stress and mitochondria-dependent pathway & Jiang et al., 2014 \\
\hline & BCG/LPS-induced immunological liver injury & 25-100 mg/kg (i.g.) & TNF- $\alpha$, IL-6, and LPS receptor & Liu et al., 2006 \\
\hline \multirow[t]{5}{*}{ Cholestasis } & ANIT-induced cholestasis & $100-200$ mg/kg (i.g.) & ROS-related NADPH and NOX4 & Zhao et al., 2013 \\
\hline & ANIT-induced cholestasis & 50-200 mg/kg (i.g.) & Apoptosis-related Bax, Caspase-9, and caspase-3 & Zhou et al., 2017 \\
\hline & ANIT-induced cholestasis & 50-200 mg/kg (i.g.) & PI3K/Akt/Nrf2 pathway & Chen Z. et al., 2015 \\
\hline & ANIT-induced cholestasis & 50-200 mg/kg (i.g.) & $\begin{array}{l}\mathrm{NF}-\kappa \mathrm{B}, \mathrm{IL}-1 \beta \text { and the hepatic transporters NTCP, BSEP, } \\
\text { and MRP2 }\end{array}$ & Zhao et al., 2017 \\
\hline & ANIT-induced cholestasis & 50-200 mg/kg (i.g.) & Primary bile acid biosynthesis & Chen et al., 2016 \\
\hline \multirow{8}{*}{$\begin{array}{l}\text { Liver } \\
\text { fibrosis }\end{array}$} & $\mathrm{CCl}_{4}$-induced liver fibrosis & 20-80 mg/kg (i.g.) & IV-C, LN, and Hyp reduction & Min et al., 2011 \\
\hline & $\mathrm{CCl}_{4}$-induced liver fibrosis & 80-200 mg/kg (i.g.) & mTOR/HIF-1 $\alpha$ signaling pathway & Zhao et al., 2014 \\
\hline & Radiation-induced liver fibrosis & 20-80 mg/kg (i.g.) & TGF- $\beta 1 /$ Smads signaling pathway & Hu et al., 2018 \\
\hline & DMN-induced liver fibrosis & 20 mg/kg (i.g.) & Macrophage disruption & Chen et al., 2012 \\
\hline & Schistosomiasis mansoni-induced liver fibrosis & 50 mg/kg (i.g.) & Apoptosis pathway related to caspase- 3 and P53 & $\begin{array}{l}\text { Abd El-Aal et al., } \\
2017\end{array}$ \\
\hline & Schistosomiasis japonica-induced liver fibrosis & 60 mg/kg (i.g.) & JAK2/STAT6 signaling pathway and IL-13 & Chu et al., 2011 \\
\hline & $\begin{array}{l}\text { Schistosomiasis japonica-induced liver fibrosis/ } \\
\text { Hepatic stellate cells }\end{array}$ & $\begin{array}{l}30 \mathrm{mg} / \mathrm{kg} \text { (i.g.) } \\
/ 30-120 \mathrm{mg} / \mathrm{L} \text { (in vitro) }\end{array}$ & SOCS-1, STAT6, and IL-13 & Li et al., 2010 \\
\hline & Schistosomiasis japonica-induced liver fibrosis & 30 mg/kg (i.g.) & IL-13 and IL-13Ra2 & Li et al., 2009 \\
\hline \multirow[t]{4}{*}{ NAFLD } & AIN76A diet-induced NAFLD & $0.05 \%$ (in diet) & Lipid synthesis, inflammation, and hyperglycemia pathway & Zhang et al., 2015 \\
\hline & HCF diet-induced NAFLD & 20-100 mg/kg (i.g.) & ROCK/NF-kB signaling pathway & Ma et al., 2016 \\
\hline & $2 \%$ cholesterol and $15 \%$ lard diet-induced NAFLD & 20 mg/kg (i.g.) & IRS/Akt/GSK3 $\beta$, antioxidation, and insulin sensitizing & Ma et al., 2017 \\
\hline & $\begin{array}{l}\text { Fructose-induced insulin resistance and hepatic } \\
\text { steatosis }\end{array}$ & $10-40$ mg/kg (i.g.) & LKB1/AMPK and Akt signaling pathway & Li et al., 2018 \\
\hline \multirow[t]{2}{*}{ HCC } & Human HCC Bel-7402 and HepG2 cell lines & $6.25-200 \mu \mathrm{M}$ (in vitro) & MMP-9, ERK, and E-cad & Lu et al., 2014 \\
\hline & Human HCC HepG2 and SMMC-7721 cell lines & $10^{-8}-10^{-5} \mathrm{~mol} / \mathrm{L}$ (in vitro) & Prostaglandin E receptor EP2, Bax, Bcl-2, and caspase-3 & Hu et al., 2013 \\
\hline
\end{tabular}

ANIT, alpha-naphthylisothiocyanate; BCG, bacillus Calmette-Guerin; $C_{C l}$, carbon tetrachloride; Con-A, concanavalin A; DMN, dimethylnitrosamine; HCC, hepatocellular carcinoma; HCF, Highfat; HHSECs, human hepatic sinusoidal endothelial cells; i.g., intragastric; I/R, ischemia/reperfusion; i.v., intravenous injection; LPS, lipopolysaccharide; NAFLD, Nonalcoholic fatty liver disease.

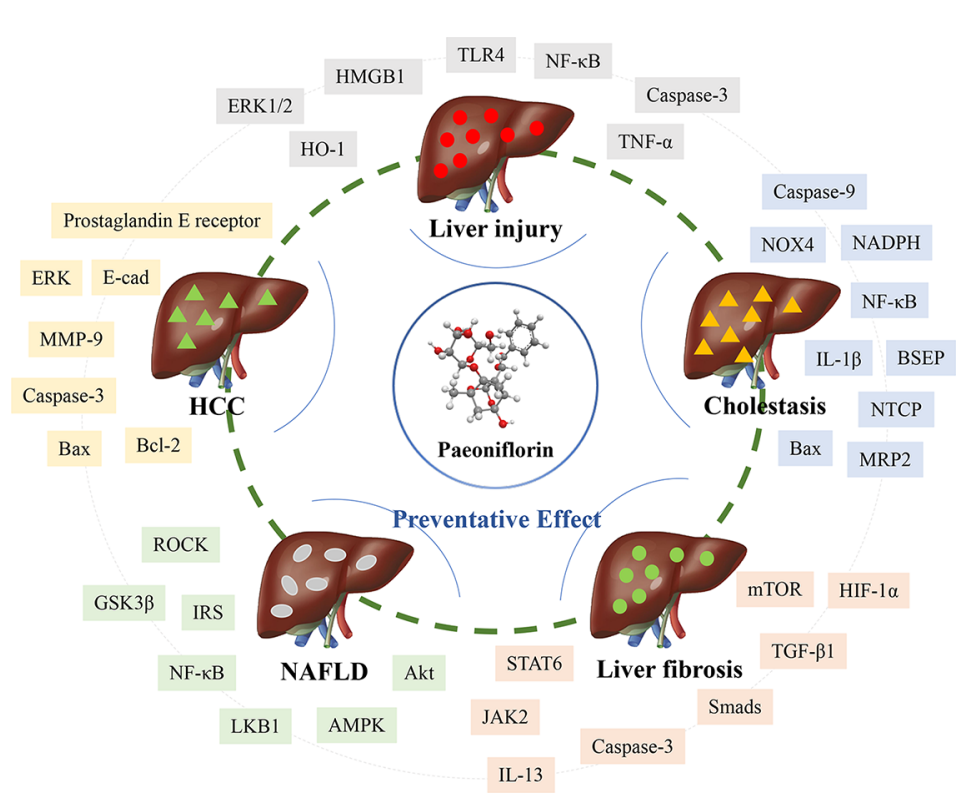

FIGURE 1 | The pharmacological effect of paeoniflorin on liver diseases through multiple targets. 
investigation. Even so, two essential aspects ought to be noted based on this mini-review. First, most of the signals mentioned above are the downstream targets in various liver diseases. The upstream of signals which paeoniflorin targets directly will be drawn with special attention. Second, particular focus should also be paid to the 'from bench to bedside' concept for further clinical discovery. The clinical conversion with rigorous randomized controlled trial is the golden index to check the efficacy and medicinal value of paeoniflorin. Therefore, the deeper mechanistic investigation and the further clinical confirmation seem as the two key processes in the future development.

In summary, paeoniflorin demonstrates multiple effects on liver diseases correlating with complex and complicated signaling pathways. Therefore, paeoniflorin might be a potential agent to treat liver disease and alleviate liver damage.

\section{REFERENCES}

Abd El-Aal, N. F., Hamza, R. S., and Harb, O. (2017). Paeoniflorin targets apoptosis and ameliorates fibrosis in murine schistosomiasis mansoni: A novel insight. Exp. Parasitol. 183, 23-32. doi: 10.1016/j.exppara.2017.10.005

Allen, K., Jaeschke, H., and Copple, B. L. (2011). Bile acids induce inflammatory genes in hepatocytes: a novel mechanism of inflammation during obstructive cholestasis. Am. J. Pathol. 178 (1), 175-186. doi: 10.1016/j.ajpath.2010.11.026

Brenner, C., Galluzzi, L., Kepp, O., and Kroemer, G. (2013). Decoding cell death signals in liver inflammation. J. Hepatol. 59 (3), 583-594. doi: 10.1016/ j.jhep.2013.03.033

Brown, G. T., and Murray, G. I. (2015). Current mechanistic insights into the roles of matrix metalloproteinases in tumour invasion and metastasis. J. Pathol. 237 (3), 273-281. doi: 10.1002/path.4586

Chen, X., Liu, C., Lu, Y., Yang, Z., Lv, Z., Xu, Q., et al. (2012). Paeoniflorin regulates macrophage activation in dimethylnitrosamine-induced liver fibrosis in rats. BMC Complement. Altern. Med. 12, 254. doi: 10.1186/1472-6882-12-254

Chen, M., Cao, L., Luo, Y., Feng, X., Sun, L., Wen, M., et al. (2015). Paeoniflorin protects against concanavalin $\mathrm{A}$-induced hepatitis in mice. Int. Immunopharmacol. 24 (1), 42-49. doi: 10.1016/j.intimp.2014.11.006

Chen, Z., Ma, X., Zhu, Y., Zhao, Y., Wang, J., Li, R., et al. (2015). Paeoniflorin ameliorates ANIT-induced cholestasis by activating Nrf2 through an PI3K/ Akt-dependent pathway in rats. Phytother. Res. 29 (11), 1768-1775. doi: $10.1002 /$ ptr.5431

Chen, Z., Zhu, Y., Zhao, Y., Ma, X., Niu, M., Wang, J., et al. (2016). Serum Metabolomic Profiling in a Rat Model Reveals Protective Function of Paeoniflorin Against ANIT Induced Cholestasis. Phytother. Res. 30 (4), 654662. doi: $10.1002 /$ ptr.5575

Chen, H., Dong, Y., He, X., Li, J., and Wang, J. (2018). Paeoniflorin improves cardiac function and decreases adverse postinfarction left ventricular remodeling in a rat model of acute myocardial infarction. Drug Des. Devel. Ther. 12, 823-836. doi: 10.2147/DDDT.S163405

Chu, D., Du, M., Hu, X., Wu, Q., and Shen, J. (2011). Paeoniflorin attenuates schistosomiasis japonica-associated liver fibrosis through inhibiting alternative activation of macrophages. Parasitology. 138 (10), 1259-1271. doi: 10.1017/ S0031182011001065

Copple, B. L., Jaeschke, H., and Klaassen, C. D. (2010). Oxidative stress and the pathogenesis of cholestasis. Semin. Liver. Dis. 30 (2), 195-204. doi: 10.1055/s0030-1253228

Estes, C., Razavi, H., Loomba, R., Younossi, Z., and Sanyal, A. J. (2018). Modeling the epidemic of nonalcoholic fatty liver disease demonstrates an exponential increase in burden of disease. Hepatology. 67 (1), 123-133. doi: 10.1002/hep.29466

Ghonem, N. S., Assis, D. N., and Boyer, J. L. (2015). Fibrates and cholestasis. Hepatol. 62 (2), 635-643. doi: 10.1002/hep.27744

Go, K. L., Lee, S., Zendejas, I., Behrns, K. E., and Kim, J. S. (2015). Mitochondrial Dysfunction and Autophagy in Hepatic Ischemia/Reperfusion Injury. Biomed. Res. Int. 2015, 183469. doi: 10.1155/2015/183469

\section{AUTHOR CONTRIBUTIONS}

$\mathrm{XM}, \mathrm{WZ}$, and YJ prepared the manuscript. JW and SW reviewed the drafts and provided important information for the completion. YZ conceived the idea and provided important information for the completion.

\section{FUNDING}

This work was supported by National Natural Science Foundation of China (81874365), Sichuan Science and Technology Program (2019YJ0492), China Postdoctoral Science Found Grant (2017M622987), and Chengdu University of TCM Found Grant (QNXZ2018025).
Gong, W. G., Lin, J. L., Niu, Q. X., Wang, H. M., Zhou, Y. C., Chen, S. Y., et al. (2015). Paeoniflorin diminishes ConA-induced IL-8 production in primary human hepatic sinusoidal endothelial cells in the involvement of ERK1/2 and Akt phosphorylation. Int. J. Biochem. Cell Biol. 62, 93-100. doi: 10.1016/ j.biocel.2015.02.017

Guo, X. Y., Yin, X. R., Yuan, H. M., and Wan, J. Y. (2018). Paeoniflorin Attenuates CCl4-Induced Acute Liver Injury. Genom. Applied. Biol. 37 (8), 3693-3698. doi: $10.13417 /$ j.gab.037.003693

Hasin, Y., Seldin, M., and Lusis, A. (2017). Multi-omics approaches to disease. Genome Biol. 18 (1), 83. doi: 10.1186/s13059-017-1215-1

Heymann, F., Peusquens, J., Ludwig-Portugall, I., Kohlhepp, M., Ergen, C., Niemietz, P., et al. (2015). Liver inflammation abrogates immunological tolerance induced by Kupffer cells. Hepatology 62 (1), 279-291. doi: 10.1002/hep.27793

Higashi, T., Friedman, S. L., and Hoshida, Y. (2017). Hepatic stellate cells as key target in liver fibrosis. Adv. Drug Deliv. Rev. 121, 27-42. doi: 10.1016/ j.addr.2017.05.007

Hu, S., Sun, W., Wei, W., Wang, D., Jin, J., Wu, J., et al. (2013). Involvement of the prostaglandin E receptor EP2 in paeoniflorin-induced human hepatoma cell apoptosis. Anticancer. Drugs 24 (2), 140-149. doi: 10.1097/CAD.0b013e32835a4dac

Hu, Z., Qin, F., Gao, S., Zhen, Y., Huang, D., and Dong, L. (2018). Paeoniflorin exerts protective effect on radiation-induced hepatic fibrosis in rats via TGFB1/Smads signaling pathway. Am. J. Transl. Res. 10 (3), 1012-1021.

Jiang, Z., Chen, W., Yan, X., Bi, L., Guo, S., and Zhan, Z. (2014). Paeoniflorin protects cells from GalN/TNF- $\alpha$-induced apoptosis via ER stress and mitochondria-dependent pathways in human L02 hepatocytes. Acta Biochim. Biophys. Sin. 46 (5), 357-367. doi: 10.1093/abbs/gmu010

Kullak-Ublick, G. A., Andrade, R. J., Merz, M., End, P., Benesic, A., Gerbes, A. L., et al. (2017). Drug-induced liver injury: recent advances in diagnosis and risk assessment. Gut 66 (6), 1154-1164. doi: 10.1136/gutjnl-2016-313369

Kunnumakkara, A. B., Bordoloi, D., Padmavathi, G., Monisha, J., Roy, N. K., Prasad, S., et al. (2017). Curcumin, the golden nutraceutical: multitargeting for multiple chronic diseases. Br. J. Pharmacol. 174 (11), 1325-1348. doi: 10.1111/ bph.13621

Lebeaupin, C., Vallée, D., Hazari, Y., Hetz, C., Chevet, E., and Bailly-Maitre, B. (2018). Endoplasmic reticulum stress signalling and the pathogenesis of non-alcoholic fatty liver disease. J. Hepatol. 69 (4), 927-947. doi: 10.1016/j.jhep.2018.06.008

Li, X., Shen, J., Zhong, Z., Wen, H., Luo, Q., and Wei, W. (2009). Paeoniflorin: a monomer from traditional Chinese medical herb ameliorates Schistosoma japonicum egg-induced hepatic fibrosis in mice. J. Parasitol. 95 (6), 1520-1524. doi: 10.1645/GE-1994.1

Li, X., Shen, J., Zhong, Z., Peng, J., Wen, H., Li, J., et al. (2010). Paeoniflorin ameliorates schistosomiasis liver fibrosis through regulating IL-13 and its signalling molecules in mice. Parasitology 137 (8), 1213-1225. doi: 10.1017/ S003118201000003X

Li, S., Tan, H. Y., Wang, N., Zhang, Z. J., Lao, L., Wong, C. W., et al. (2015). The Role of Oxidative Stress and Antioxidants in Liver Diseases. Int. J. Mol. Sci. 16 (11), 26087-26124. doi: 10.3390/ijms161125942 
Li, Y. C., Qiao, J. Y., Wang, B. Y., Bai, M., Shen, J. D., and Cheng, Y. X. (2018). Paeoniflorin Ameliorates Fructose-Induced Insulin Resistance and Hepatic Steatosis by Activating LKB1/AMPK and AKT Pathways. Nutrients 10 (8), E1024. doi: 10.3390/nu10081024

Liu, D. F., Wei, W., and Song, L. H. (2006). Protective effect of paeoniflorin on immunological liver injury induced by bacillus Calmette-Guerin plus lipopolysaccharide: modulation of tumour necrosis factor-alpha and interleukin-6 MRNA. Clin. Exp. Pharmacol. Physiol. 33 (4), 332-339. doi: 10.1111/j.1440-1681.2006.04371.x

Lu, J. T., He, W., Song, S. S., and Wei, W. (2014). Paeoniflorin inhibited the tumor invasion and metastasis in human hepatocellular carcinoma cells. Bratisl. Lek. Listy. 115 (7), 427-433. doi: 10.4149/BLL_2014_084

Ma, Z., Chu, L., Liu, H., Li, J., Zhang, Y., Liu, W., et al. (2016). Paeoniflorin alleviates non-alcoholic steatohepatitis in rats: Involvement with the ROCK/ NF- $\mathrm{KB}$ pathway. Int. Immunopharmacol. 38, 377-384. doi: 10.1016/ j.intimp.2016.06.023

Ma, Z., Chu, L., Liu, H., Wang, W., Li, J., Yao, W., et al. (2017). Beneficial effects of paeoniflorin on non-alcoholic fatty liver disease induced by high-fat diet in rats. Sci. Rep. 7, 44819. doi: 10.1038/srep44819

Ma, X., Wen, J. X., Gao, S. J., He, X., Li, P. Y., Yang, Y. X., et al. (2018). Paeonia lactiflora Pall. regulates the NF- $\kappa \mathrm{B}-\mathrm{NLRP} 3$ inflammasome pathway to alleviate cholestasis in rats. J. Pharm. Pharmacol. 70 (12), 1675-1687. doi: 10.1111/ jphp.13008

Ma, X., Jiang, Y., Zhang, W., Wang, J., Wang, R., Wang, L., et al. (2020). Natural products for the prevention and treatment of cholestasis: A review. Phytother. Res. doi: 10.1002/ptr.6621

Min, J., Han, Z., and Jing, C. (2011). Protective effect of paeoniflorin on rat with chronic liver injury and fibrosis. Modern. J. Integrated. Tradit. Chin. Western Med. 20 (36), 4618-4624.

Neuschwander-Tetri, B. A., Clark, J. M., Bass, N. M., Van Natta, M. L., UnalpArida, A., Tonascia, J., et al. (2010). Clinical, laboratory and histological associations in adults with nonalcoholic fatty liver disease. Hepatology 52 (3), 913-924. doi: 10.1002/hep.23784

Park, E. J., and Pezzuto, J. M. (2015). The pharmacology of resveratrol in animals and humans. Biochim. Biophys. Acta 1852 (6), 1071-1113. doi: 10.1016/ j.bbadis.2015.01.014

Peralta, C., Jiménez-Castro, M. B., and Gracia-Sancho, J. (2013). Hepatic ischemia and reperfusion injury: effects on the liver sinusoidal milieu. J. Hepatol. 59 (5), 1094-1106. doi: 10.1016/j.jhep.2013.06.017

Ren, J., Fu, L., Nile, S. H., Zhang, J., and Kai, G. (2019). Salvia miltiorrhiza in Treating Cardiovascular Diseases: A Review on Its Pharmacological and Clinical Applications. Front. Pharmacol. 10, 753. doi: 10.3389/ fphar.2019.00753

Robinson, A., Tavakoli, H., Cheung, R., Liu, B., Bhuket, T., and Wong, R. J. (2019). Low Rates of Retention Into Hepatocellular Carcinoma (HCC) Surveillance Program After Initial HCC Screening. J. Clin. Gastroenterol. 53 (1), 65-70. doi: 10.1097/MCG.0000000000001024

Seki, E., and Brenner, D. A. (2015). Recent advancement of molecular mechanisms of liver fibrosis. J. Hepatobiliary. Pancreat. Sci. 22 (7), 512-518. doi: 10.1002/ jhbp. 245

Seki, E., and Schwabe, R. F. (2015). Hepatic inflammation and fibrosis: functional links and key pathways. Hepatology 61 (3), 1066-1079. doi: 10.1002/hep.27332

Singh, D., Cho, W. C., and Upadhyay, G. (2016). Drug-Induced Liver Toxicity and Prevention by Herbal Antioxidants: An Overview. Front. Physiol. 6, 363. doi: 10.3389/fphys.2015.00363

Su, L., Zhou, T., Zhang, Z., Zhang, X., Zhi, X., Li, C., et al. (2016). Optimal staging system for predicting the prognosis of patients with hepatocellular carcinoma in China: a retrospective study. BMC Cancer. 16, 424. doi: 10.1186/s12885-0162420-0

Tao, Y. E., Wen, Z., Song, Y., and Wang, H. (2016). Paeoniflorin attenuates hepatic ischemia/reperfusion injury via anti-oxidative, anti-inflammatory and antiapoptotic pathways. Exp. Ther. Med. 11 (1), 263-268. doi: 10.3892/ etm.2015.2902

Trauner, M., Fuchs, C. D., Halilbasic, E., and Paumgartner, G. (2017). New therapeutic concepts in bile acid transport and signaling for management of cholestasis. Hepatology 65 (4), 1393-1404. doi: 10.1002/hep.28991
Tsuchida, T., and Friedman, S. L. (2017). Mechanisms of hepatic stellate cell activation. Nat. Rev. Gastroenterol. Hepatol. 14 (7), 397-411. doi: 10.1038/ nrgastro. 2017.38

Tu, J., Guo, Y., Hong, W., Fang, Y., Han, D., Zhang, P., et al. (2019). The regulatory effects of Paeoniflorin and its derivative Paeoniflorin-6'-O-benzene sulfonate CP-25 on inflammation and immune diseases. Front. Pharmacol. 10, 57. 2019. doi: 10.3389/fphar.2019.00057

Wang, S., Pacher, P., De,, Lisle, R. C., Huang, H., and Ding, W. X. (2016). A Mechanistic Review of Cell Death in Alcohol-Induced Liver Injury. Alcohol. Clin. Exp. Res. 40 (6), 1215-1223. doi: 10.1111/acer.13078

Wree, A., McGeough, M. D., Inzaugarat, M. E., Eguchi, A., Schuster, S., Johnson, C. D., et al. (2018). NLRP3 inflammasome driven liver injury and fibrosis: Roles of IL-17 and TNF in mice. Hepatology 67 (2), 736-749. doi: 10.1002/ hep. 29523

Xie, T., Li, K., Gong, X., Jiang, R., Huang, W., Chen, X., et al. (2018). Paeoniflorin protects against liver ischemia/reperfusion injury in mice via inhibiting HMGB1-TLR4 signaling pathway. Phytother. Res. 32 (11), 2247-2255. doi: 10.1002/ptr.6161

Zhai, W., Ma, Z., Wang, W., Song, L., and Yi, J. (2018). Paeoniflorin inhibits Rho kinase activation in joint synovial tissues of rats with collagen-induced rheumatoid arthritis. Biomed. Pharmacother. 106, 255-259. doi: 10.1016/ j.biopha.2018.06.130

Zhang, L., Yang, B., and Yu, B. (2015). Paeoniflorin Protects against Nonalcoholic Fatty Liver Disease Induced by a High-Fat Diet in Mice. Biol. Pharm. Bull. 38 (7), 1005-1011. doi: 10.1248/bpb.b14-00892

Zhang, Y., Qiao, L., Xu, W., Wang, X., Li, H., Xu, W., et al. (2017). Paeoniflorin Attenuates Cerebral Ischemia-Induced Injury by Regulating $\mathrm{Ca}^{2+} / \mathrm{CaMKII} /$ CREB Signaling Pathway. Molecules 22 (3), E359. doi: 10.3390/ molecules22030359

Zhang, H. Y., Wang, H. L., Zhong, G. Y., and Zhu, J. X. (2018). Molecular mechanism and research progress on pharmacology of traditional Chinese medicine in liver injury. Pharm. Biol. 56 (1), 594-611. doi: 10.1080/ 13880209.2018.1517185

Zhang, J., Yu, K., Han, X., Zhen, L., Liu, M., Zhang, X., et al. (2018). Paeoniflorin influences breast cancer cell proliferation and invasion via inhibition of the Notch-1 signaling pathway. Mol. Med. Rep. 17 (1), 1321-1325. doi: 10.3892/ mmr.2017.8002

Zhao, Y., Zhou, G., Wang, J., Jia, L., Zhang, P., Li, R., et al. (2013). Paeoniflorin protects against ANIT-induced cholestasis by ameliorating oxidative stress in rats. Food Chem. Toxicology 58, 242-248. doi: 10.1016/j.fct.2013.04.030

Zhao, Y., Ma, X., Wang, J., Zhu, Y., Li, R., Wang, J., et al. (2014). Paeoniflorin alleviates liver fibrosis by inhibiting HIF-1 $\alpha$ through mTOR-dependent pathway. Fitoterapia 99, 318-327. doi: 10.1016/j.fitote.2014.10.009

Zhao, D. D., Jiang, L. L., Li, H. Y., Yan, P. F., and Zhang, Y. L. (2016). Chemical Components and Pharmacological Activities of Terpene Natural Products from the Genus Paeonia. Molecules 21 (10), E1362. doi: 10.3390/ molecules 21101362

Zhao, Y., He, X., Ma, X., Wen, J., Li, P., Wang, J., et al. (2017). Paeoniflorin ameliorates cholestasis via regulating hepatic transporters and suppressing inflammation in ANIT-fed rats. Biomed. Pharmacother. 89, 61-68. doi: 10.1016/j.biopha.2017.02.025

Zhou, H. Q., Liu, W., Wang, J., Huang, Y. Q., Li, P. Y., Zhu, Y., et al. (2017). Paeoniflorin attenuates ANIT-induced cholestasis by inhibiting apoptosis in vivo via mitochondria-dependent pathway. Biomed. Pharmacother. 89, 696704. doi: 10.1016/j.biopha.2017.02.084

Conflict of Interest: The authors declare that the research was conducted in the absence of any commercial or financial relationships that could be construed as a potential conflict of interest.

Copyright (c) $2020 \mathrm{Ma}$, Zhang, Jiang, Wen, Wei and Zhao. This is an open-access article distributed under the terms of the Creative Commons Attribution License (CC BY). The use, distribution or reproduction in other forums is permitted, provided the original author(s) and the copyright owner(s) are credited and that the original publication in this journal is cited, in accordance with accepted academic practice. No use, distribution or reproduction is permitted which does not comply with these terms. 